\title{
Effect of Batch and Fed-Batch Growth Modes on Biofilm Formation by Listeria monocytogenes at Different Temperatures
}

\author{
Diana A. Rodrigues $\cdot$ Marta A. Almeida • \\ Pilar A. Teixeira $\cdot$ Rosário T. Oliveira . \\ Joana C. Azeredo
}

Received: 20 April 2009/Accepted: 8 July 2009/Published online: 4 August 2009

(C) Springer Science+Business Media, LLC 2009

\begin{abstract}
The influence of Listeria monocytogenes ( $L$. monocytogenes) biofilm formation feeding conditions (batch and fed-batch) at different temperatures on biofilm biomass and activity was determined. Biofilm biomass and cellular metabolic activity were assessed by Crystal Violet (CV) staining and 2,3-bis(2-methoxy-4-nitro-5-sulfophenyl)-2H-tetrazolium-5-carboxanilide inner salt (XTT) colorimetric method, respectively. Live/Dead staining was also performed in order to get microscopic visualization of the different biofilms. Results revealed that at refrigeration temperature $\left(4^{\circ} \mathrm{C}\right)$ a higher amount of biofilm was produced when batch conditions were applied, while at higher temperatures the fed-batch feeding condition was the most effective on biofilm formation. Moreover, independently of the temperature used, biofilms formed under fed-batch conditions were metabolically more active than those formed in batch mode. In conclusion, this work shows that different growth modes significantly influence L. monocytogenes biofilm formation on abiotic surfaces as well as the metabolic activity of cells within biofilms.
\end{abstract}

\section{Introduction}

Several studies have already been done regarding adhesion and biofilm formation by different Listeria monocytogenes strains [3, 5, 11]. However, it is important to notice that

D. A. Rodrigues - M. A. Almeida · P. A. Teixeira

R. T. Oliveira - J. C. Azeredo $(\square)$

IBB - Institute for Biotechnology and Bioengineering, Centre of

Biological Engineering, University of Minho, Campus de

Gualtar, 4710-057 Braga, Portugal

e-mail: jazeredo@deb.uminho.pt final conclusions about biofilm formation capability, exopolysaccharide production and biofilms viability, among others, may differ not only due to differences between specific strains tested $[3,12]$ but also because of the different methods and conditions applied in each work [3, 6, $11,17]$. Until now, some of the most studied parameters involved in biofilm formation by L. monocytogenes have been: medium composition [1, 17], material surfaces [16, 19], incubation temperature [8, 16], and incubation time [11]. However, even though researchers seem to arbitrarily choose batch or fed-batch conditions to assess biofilm formation by L. monocytogenes [5, 8, 11], to our knowledge nothing is known on the effect of these two growth modes on such biological process.

In this work, biofilm formation by five L. monocytogenes strains was assessed under batch and fed-batch conditions at three different temperatures $\left(4,25\right.$, and $\left.37^{\circ} \mathrm{C}\right)$ in order to evaluate how these distinct growth modes might interfere with biofilm development on an abiotic surface, in terms of biomass and cells' viability.

\section{Materials and Methods}

\section{Bacterial Strains and Culture Conditions}

All assays were performed with five L. monocytogenes strains: 747, 925, 930, and 994 are food isolates belonging to distinct serotypes-747, 925 and 930 present serotype 1/ 2b, while strain 994 presents serotype 4ab-whereas 1562 is a clinical isolate presenting serotype $4 \mathrm{~b}$. All strains were kindly provided by Dr. Paula Teixeira (Escola Superior de Biotecnologia, Universidade Católica Portuguesa, Porto, Portugal). For each assay, strains were subcultured on trypticase soy agar (TSA; Merck, Germany) for 24-48 h at 
$37^{\circ} \mathrm{C}$ and then grown in $30 \mathrm{ml}$ of tryptic soy broth (TSB, Merck, Germany) for $18 \pm 2 \mathrm{~h}$ at room temperature with agitation at $120 \mathrm{rpm}$. Cells were harvested by centrifugation $\left(5 \mathrm{~min}, 9000 \mathrm{rpm}, 22^{\circ} \mathrm{C}\right)$, washed twice with sterile phosphate-buffered saline (PBS $0.1 \mathrm{M}, \mathrm{pH} 7$ ) and cell suspensions were standardized to an optical density $\left(\mathrm{OD}_{640 \mathrm{~nm}}\right) \approx 0.3$ corresponding to a concentration of approximately $1 \times 10^{9} \mathrm{CFU} \mathrm{ml}^{-1}$.

\section{Biofilm Formation in Fed-Batch Mode}

Biofilm formation assays were performed in sterile 96-well flat-bottomed uncoated polystyrene tissue culture plates (Orange Scientific, Belgium). Each well was filled with $240 \mu \mathrm{l}$ of TSB supplemented with $0.25 \%$ (w/v) of glucose (Merck) and $10 \mu \mathrm{l}$ of cell suspension. Negative controls consisted of wells filled only with culture medium without any bacterial cells. The plates were incubated at 4,25 , and $37^{\circ} \mathrm{C}$, for 5 days, with constant agitation at $120 \mathrm{rpm}$. The culture medium was refreshed twice a day by carefully pipetting $240 \mu \mathrm{l}$ of each well (with care not to touch the bottom and the sides of the well) and gently adding the same volume of fresh medium. Four independent assays were performed for each strain at each condition with eight wells per strain per assay.

\section{Biofilm Formation in Batch Mode}

Biofilms were formed on microtiter plates as described above, except that there was no replacement of medium during all the incubation period.

\section{Determination of Biofilm Biomass}

Biofilm biomass was assessed as previously described [9] with some modifications. Briefly, at each time settled to stop and analyze the biofilms formed medium was removed by pipetting and each well washed with PBS, also by pipetting. Biofilms were then fixed with $200 \mu \mathrm{l}$ of methanol (Merck, Germany) per well for $15 \mathrm{~min}$. Following, the liquid phase was removed and the plates were left to dry at room temperature until they were completely dehydrated. Biofilm in each well was then stained with $200 \mu \mathrm{l}$ of an aqueous $1 \%(\mathrm{w} / \mathrm{v}) \mathrm{CV}$ solution (Merck, Germany) for $5 \mathrm{~min}$ at room temperature, and the excess dye rinsed off by washing with PBS. Once again, the plates were left at room temperature until a complete drying was achieved. The dye bound to biofilms in each well was resolubilized with $200 \mu \mathrm{l}$ of $33 \%$ (v/v) acetic acid (Merck, Germany) and the optic density (OD) of each well measured at $570 \mathrm{~nm}$ in a microplate reader $\left(\mathrm{BIO}-\mathrm{TEK}^{\circledR}\right.$ Synergy HT, IZASA Portugal).
Determination of Cellular Metabolic Activity

Cellular metabolic activity was assessed by the reduction of tetrazolium salt (XTT) as described previously [13] with some modifications. Briefly, biofilms were gently washed with PBS and then $250 \mu \mathrm{l}$ of an aqueous solution containing $50 \mu \mathrm{g} \mathrm{ml}^{-1}$ XTT (Sigma, MO, USA) and $10 \mu \mathrm{g} \mathrm{ml}^{-1}$ phenazine methosulphate (PMS; Sigma, MO, USA) was added to each well. Microtiter plates were incubated for $3 \mathrm{~h}$ at $37^{\circ} \mathrm{C}$ in the dark and the OD measured at $490 \mathrm{~nm}$. Ratio $\left(\mathrm{OD}_{490 \mathrm{~nm}} / \mathrm{OD}_{570 \mathrm{~nm}}\right)$ was calculated in order to evaluate cell activity per biofilm biomass.

\section{Epifluorescence Microscopy}

In order to get microscopic observations of cell's viability, biofilms were formed on polystyrene coupons under the same batch and fed-batch conditions described above. After 5 days of incubation, coupons were carefully washed with PBS, mounted on a glass slide and stained with Live/ Dead (L/D) Backlight Kit (Molecular Probes, Eugene, OR). The two reagents (syto9 and propidium iodide) were prepared according to the manufacture's instructions and mixed in equal proportions. The mixture $(50 \mu \mathrm{l}$ per coupon) was then applied to each coupon and incubated for $15 \mathrm{~min}$ in the dark. Biofilms were visualized under an epifluorescence microscope (Olympus BX 51) equipped with a filter block that simultaneously detects the two components of the mixture.

\section{Statistical Analysis}

The statistical analysis was performed using the statistical program SPSS (Statistical Package for the Social Sciences). The results were compared using the non-parametric Mann-Whitney $U$-test at a $95 \%$ confidence level.

\section{Results}

\section{Biofilms Biomass}

The analysis of the effect of distinct growth modes on $L$. monocytogenes biofilm formation on polystyrene showed different performances for batch and fed-batch conditions, since at refrigeration temperature (Fig. 1a) batch conditions lead to greater biomass amounts than fed-batch conditions, while at higher temperatures (Fig. 1b, c) it was the fed-batch mode the most effective on enhancing biofilm formation $(P<0.05)$. Although not easily seen in the figures, it is worth to notice that for most strains biofilms grown under batch conditions had a general decrease of $\mathrm{OD}_{570 \mathrm{~nm}}$ values at 25 and $37^{\circ} \mathrm{C}$ between the $3 \mathrm{rd}$ and 4 th 
Fig. 1 Biofilm formation measured by crystal violet staining on $\square$ batch mode and $\therefore:$ fed-batch mode at a $4, \mathbf{b} 25$, and c $37^{\circ} \mathrm{C}$. Bars represent average $\mathrm{CV}-\mathrm{OD}_{570}$ values and standard errors. Each pair of bars represents one strain, from left to right: $747,925,930,994$, and 1562. Symbols indicate statistically different values $(P<0.05)$ within each strain considering different growth modes (*) and between strains considering the same growth mode $(\dagger)$
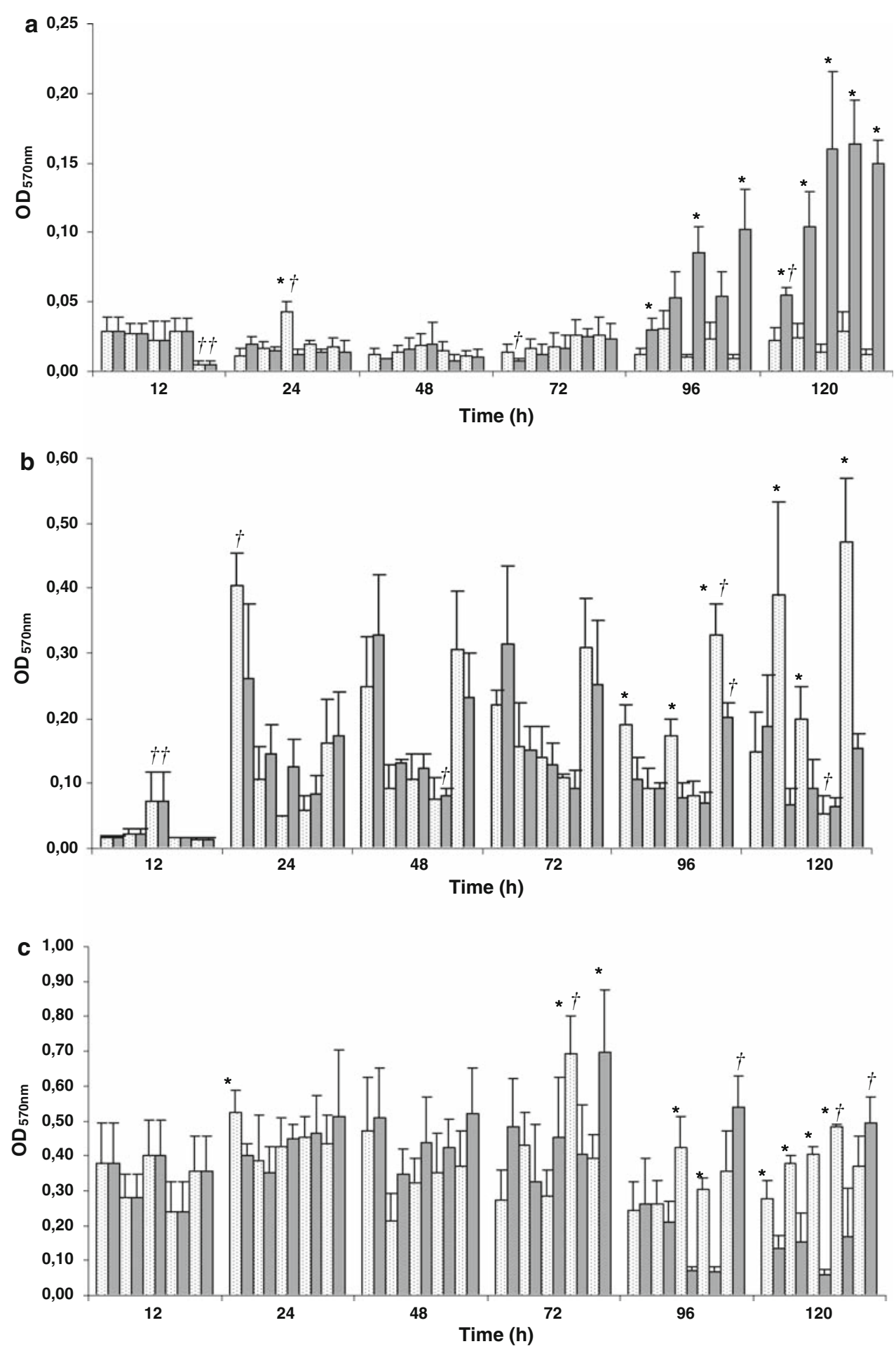

day, the same period when biofilms formed under batch mode at refrigeration temperature achieved a significant biomass increase for most strains.

\section{Cellular Metabolic Activity}

Concerning the effect of distinct growth modes on biofilms' metabolic activity, and despite few exceptions, biofilms formed under fed-batch conditions were significantly more active than biofilms formed under batch conditions, independently of temperature or incubation time (Fig. 2). This was corroborated by the microscopy images obtained after L/D staining, where biofilms formed under fed-batch mode (Fig. 3a, c) exhibit more green cellswhich indicates that most cells have an unaltered cell membrane integrity_-while biofilms formed under batch 
Fig. 2 Biofilms cellular activity estimated by $\left(\mathrm{OD}_{490 \mathrm{~nm}} /\right.$ $\left.\mathrm{OD}_{570 \mathrm{~nm}}\right)$ ratio on $\square$ batch mode and $::$ fed-batch mode at a 4, b 25 , and c $37^{\circ} \mathrm{C}$. Bars represent average $\left(\mathrm{OD}_{490 \mathrm{~nm}} /\right.$ $\left.\mathrm{OD}_{570 \mathrm{~nm}}\right)$ values and standard errors. Each pair of bars represents one strain, from left to right: $747,925,930,994$, and 1562. Symbol $*$ indicates significantly different values $(P<0.05)$ within each strain considering different growth modes
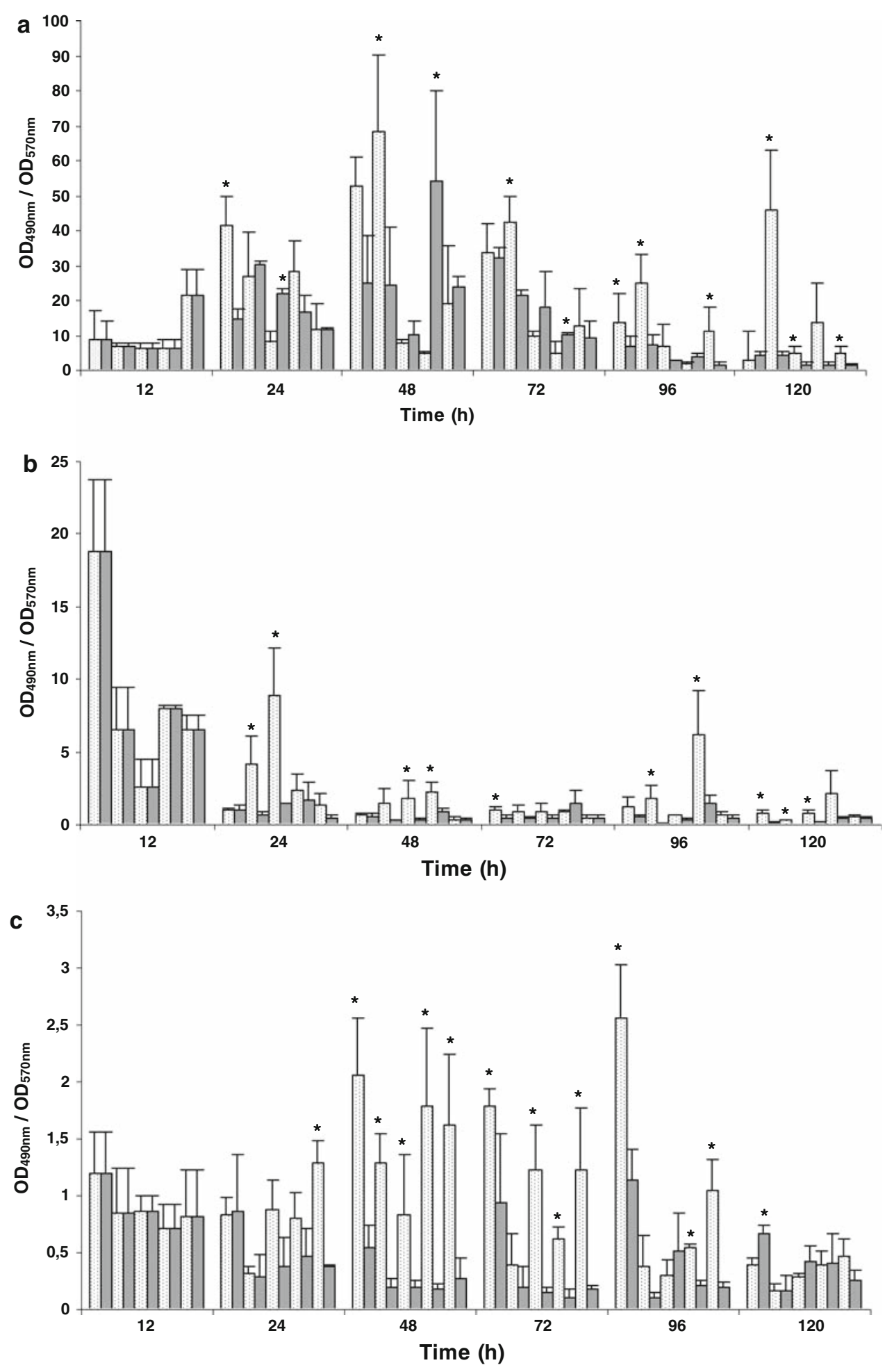

conditions (Fig. 3b, d) present more red cells-which indicates that most cells have a damaged membrane. It is also worth noting that, in contrast to what was observed in biomass assays, metabolic activity results were significantly lower $(P<0.05)$ at 25 and $37^{\circ} \mathrm{C}$ comparing to the values found at refrigeration temperature (Fig. 2).

\section{Discussion}

A general overview of the data obtained with both growth modes revealed that incubation temperature played a crucial role in L. monocytogenes biofilm development on polystyrene. It is worth noting that the highest biomass 
Fig. 3 Visualization of metabolically active cells by epifluorescence microscopy on 5-days-old L. monocytogenes biofilms formed on polystyrene coupons under fed-batch (a) and batch mode (b) at $4^{\circ} \mathrm{C}$, and under fed-batch (c) and batch mode (d) at $37^{\circ} \mathrm{C}$. Pictures were taken under a $40 \times$ objective after L/D staining
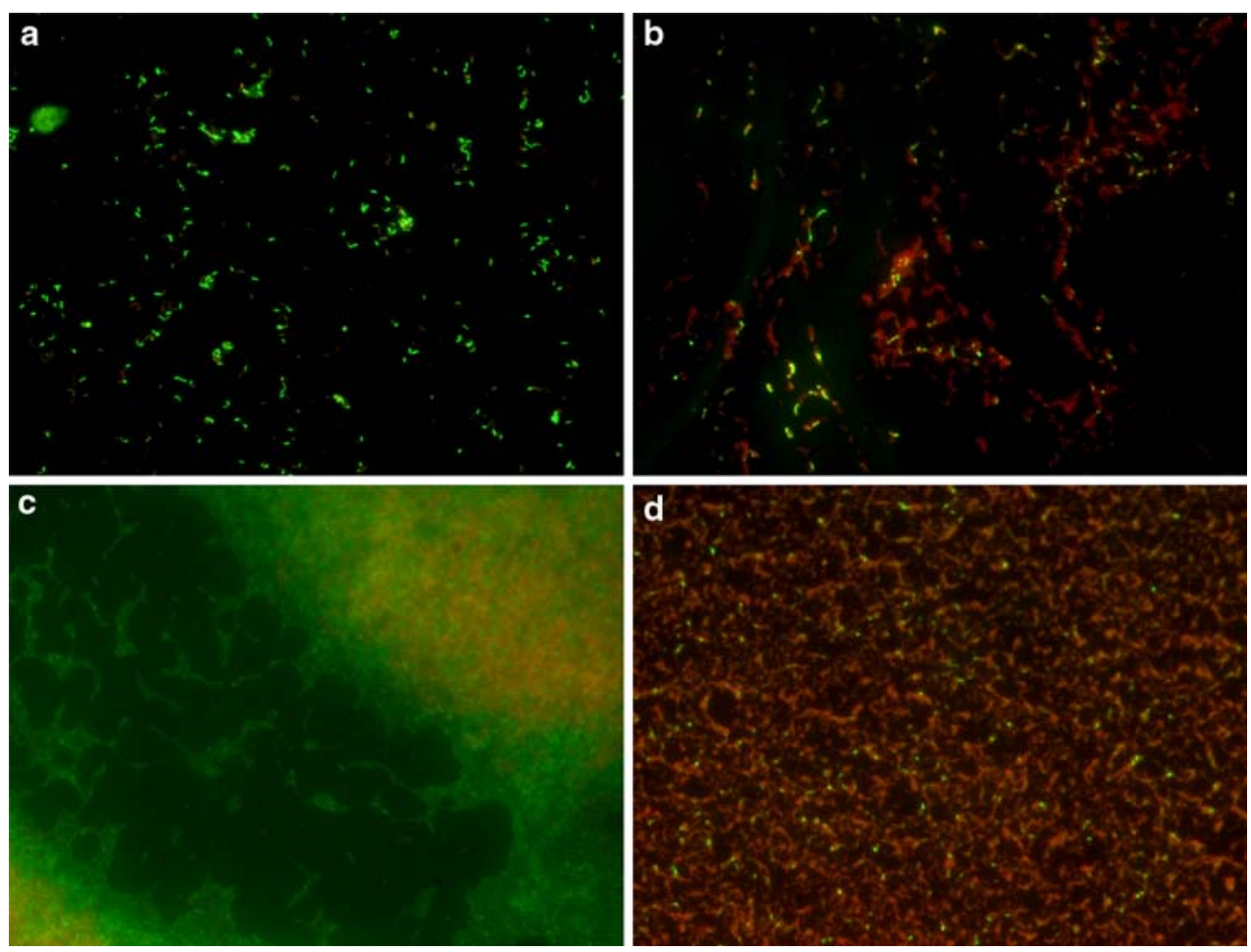

amount developed at $37^{\circ} \mathrm{C}$ is in agreement with works that showed that L. monocytogenes produces more biofilm as temperature increases $[8,14,15,19]$. On the other hand, apart from optimal growth temperature L. monocytogenes is also able to survive over a wide range of temperatures including refrigeration ones $\left(2-4^{\circ} \mathrm{C}\right)$, as it was confirmed in this work by the significantly high $\mathrm{OD}_{490 \mathrm{~nm}} / \mathrm{OD}_{570 \mathrm{~nm}}$ values observed in biofilms formed at $4^{\circ} \mathrm{C}$ (Fig. 2a) and the microscopy images (Fig. 3). This means that, although at this temperature a low amount of biomass is formed, cells within the biofilms are metabolically more active than those of biofilms formed at $37^{\circ} \mathrm{C}$. The fact that biofilms formed at $4^{\circ} \mathrm{C}$ presented low biomass values can be due to a bacterial slow growth and a low accumulation of exopolymers. Indeed, quantification of total exopolysaccharides by Dubois method [10], after matrix extraction by sonication, showed that biofilms formed at refrigeration temperature did not have a detectable amount of polysaccharides and only biofilms formed at $37^{\circ} \mathrm{C}$ under fed-batch conditions showed to have some polysaccharides in their matrix (data not shown). Moreover, the epifluorescence images (Fig. 3b) are in agreement with Bonaventura et al. [2] work in which it was reported that biofilms formed on polystyrene at $4^{\circ} \mathrm{C}$ (in batch condition) consisted of sparse clusters of cells with minimum amounts of exopolymers. The results obtained are also in accordance with Chavant et al. [8] work in which it they assessed L. monocytogenes adhesion and biofilm formation on polytetrafluoroethylene (a hydrophobic surface as polystyrene) under fed-batch conditions at three temperatures $\left(8,20\right.$, and $\left.37^{\circ} \mathrm{C}\right)$ and had found that at the lowest temperature the colonization of the surface was very slow and no bacterial mat could be formed. In that same work, the researchers concluded that the nature of the surface (hydrophobicity) and the temperature were main factors which significantly affected adhesion and biofilm formation.

Considering biomass results for each growth mode, the differences found reflect how biofilms react to environments with different amounts of available nutrients. In fact, biofilms grown at higher temperatures seem to have higher growth rates (attested by their high biomass levels) and, thus, must demand a larger amount of nutrients available. So, although cells under batch mode at 25 and $37^{\circ} \mathrm{C}$ had managed to grow in the first days, the growing biomass amount together with the lack of nutrients might have caused biofilms' deterioration and/or detachment. This deterioration can also be responsible for the low $\left(\mathrm{OD}_{490 \mathrm{~nm}} /\right.$ $\mathrm{OD}_{570 \mathrm{~nm}}$ ) values (Fig. 2b, c). Previous studies have showed that restrictions in essential nutrients occurring in solid structures may result in a considerable decrease in bacterial metabolic activity [7, 18], which is in agreement with the microscopy images obtained in this work, where the large amount of red cells on biofilm formed at $37^{\circ} \mathrm{C}$ under batch conditions is a clear sign of cells' membrane damage (Fig. 3d). On the other hand, and as stated above, cells at refrigeration temperatures display a slow growth, produce lower amount of exopolymers, and need longer adaptation periods to start growing. So, unlike what may happen in 
fed-batch mode, in which loosely adhered cells may be washed out every time the medium is refreshed [4], in batch conditions cells remain in the system and, despite the slow growth, a higher amount of biomass might be accumulated.

Concluding, in long term assays (longer than 2 days) fed-batch conditions were the most prone to promote biofilm formation by L. monocytogenes on polystyrene when high incubation temperatures are used, while in a refrigerated environment it was batch mode that enhanced a higher biomass formation. It is worth noting that the growth mode applied also affects the metabolic activity of cells within biofilms, since fed-batch mode lead to biofilms metabolically more active at all temperatures. So, when assessing biofilm formation by L. monocytogenes strains on such abiotic surfaces, researchers must take into consideration that different growth modes do lead to divergent results determining the extent to which a strain will produce biofilm and influencing the metabolic activity of biofilms' constituent cells.

Acknowledgments Authors gratefully acknowledge the helpful contribution of Lúcia Simões on the microscopy assays. This work was supported by Portuguese Science Foundation (FCT) through the project PPCDT/AGR/59358/2004. Diana Rodrigues and Pilar Teixeira acknowledge the financial support of FCT through Grants SFRH/ BD/28887/2006 and SFRH/BPD/26803/2006, respectively.

\section{References}

1. Asperger $\mathrm{H}$, Heistinger $\mathrm{H}$, Wagner $\mathrm{M}$ et al (1999) A contribution of Listeria enrichment methodology - growth of Listeria monocytogenes under varying conditions concerning enrichment broth composition, cheese matrices and competing microbial flora. Food Microbiol 16:419-431

2. Bonaventura GD, Piccolomini R, Paludi D et al (2008) Influence of temperature on biofilm formation by Listeria monocytogenes on various food-contact surfaces: relationship with motility and cell surface hydrophobicity. J Appl Microbiol 104:1552-1561

3. Borucki MK, Peppin JD, White D et al (2003) Variation in biofilm formation among strains of Listeria monocytogenes. Appl Environ Microbiol 69:7336-7342

4. Cerca N, Pier GB, Oliveira R et al (2004) Comparative evaluation of coagulase-negative staphylococci (CoNS) adherence to acrylic by a static method and a parallel-plate flow dynamic method. Res Microbiol 155:755-760

5. Chae MS, Schraft H (2000) Comparative evaluation of adhesion and biofilm formation of different Listeria monocytogenes strains. Int J Food Microbiol 62:103-111

6. Chae MS, Schraft H (2001) Cell viability of Listeria monocytogenes biofilms. Food Microbiol 18:103-112

7. Chapman AG, Fall L, Atkinson DE (1971) Adenylate energy charge in Escherichia coli during growth and starvation. J Bacteriol 108:1072-1086

8. Chavant P, Martinie B, Meylheuc T et al (2002) Listeria monocytogenes LO28: surface physicochemical properties and ability to form biofilms at different temperatures and growth phases. Appl Environ Microbiol 68:728-737

9. Djordjevic D, Wiedmann M, McLandsborough LA (2002) Microtiter plate assay for assessment of Listeria monocytogenes biofilm formation. Appl Environ Microbiol 68:2950-2958

10. Dubois M, Gilles KA, Hamilton JK et al (1956) Colorimetric method for determination of sugars and related substances. Anal Chem 28:350-355

11. Harvey J, Keenan KP, Gilmour A (2007) Assessing biofilm formation by Listeria monocytogenes strains. Food Microbiol 24:380-392

12. Kalmokoff ML, Austin JW, Wan X-D et al (2001) Adsorption, attachment and biofilm formation among isolates of Listeria monocytogenes using model conditions. J Appl Microbiol 91:725-734

13. Logu A, Pellerano M, Sanna A (2003) Comparison of the susceptibility testing of clinical isolates of Mycobacterium tuberculosis by the XTT colorimetric method and the NCCLS standards method. Int J Antimicrob Agents 21:244-250

14. Møretrø T, Langsrud S (2004) Listeria monocytogenes: biofilm formation and persistence in food-processing environments. Biofilms 1:107-121

15. Norwood DE, Gilmour A (2001) The differential adherence capabilities of two Listeria monocytogenes strains in monoculture and multispecies biofilms as a function of temperature. Lett Appl Microbiol 33:320-324

16. Sinde E, Carballo J (2000) Attachment of Salmonella spp. and Listeria monocytogenes to stainless steel, rubber and polytetrafluoroethylene: the influence of free energy and the effect of commercial sanitizers. Food Microbiol 17:439-447

17. Stepanović S, Circović I, Ranin L et al (2004) Biofilm formation by Salmonella spp. and Listeria monocytogenes on plastic surface. Lett Appl Microbiol 38:428-432

18. Walker SL, Brocklehurst TF, Wimpenny JWT (1998) Adenylates and adenylate-energy charge in submerged and planktonic cultures of Salmonella enteritidis and Salmonella typhimurium. Int J Food Microbiol 44:107-113

19. Wong ACL (1998) Biofilms in food processing environments. J Dairy Sci 81:2765-2770 